\title{
The neutrino filter: connecting blazars with ultra high energy cosmic rays and astrophysical neutrinos
}

\author{
E.Resconi ${ }^{1}$, P. Padovani ${ }^{2,3}$, S. Coenders ${ }^{1}$, A. Turcati ${ }^{1}$, P. Giommi ${ }^{4,5}$, L. Caccianiga ${ }^{6}$ \\ ${ }^{1}$ Technische Universität München, Physik-Department, James-Frank-Str. 1, D-85748 Garching \\ bei München, Germany \\ ${ }^{2}$ European Southern Observatory, Karl-Schwarzschild-Str. 2, D-85748 Garching bei München, \\ Germany \\ ${ }^{3}$ Associated to INAF - Osservatorio Astronomico di Roma, via Frascati 33, I-00040 Monteporzio \\ Catone, Italy \\ ${ }^{4}$ Italian Space Agency, ASI, via del Politecnico s.n.c., I-00133 Roma Italy \\ ${ }^{5}$ ICRANet-Rio, CBPF, Rua Dr. Xavier Sigaud 150, 22290-180 Rio de Janeiro, Brazil \\ ${ }^{6}$ INFN sezione di Milano, Dipartimento di Fisica, Universitá degli Studi di Milano via Celoria \\ 16, 20133 Milano, Italy \\ E-mail: elisa.resconi@tum.de*
}

\begin{abstract}
We discuss the link between high energy $\gamma$-ray emitting blazars, very high energy neutrinos, and ultra high energy cosmic rays using the IceCube neutrinos as a filter to gamma-ray catalogues. The neutrino filtered emitters are investigated through a correlation analysis based on a likelihood method with the ultra high energy cosmic rays from the Pierre Auger Observatory and the Telescope Array by scanning in flux and angular separation between sources and cosmic rays. Based on the presently published neutrino and cosmic ray catalogues, a 2.9 sigma excess of cosmic rays correlating with the neutrino filtered emitters is observed. The neutrino filtered $\gamma$-ray sources that make up the cosmic rays excess are blazars of the high synchrotron peak type. More statistics is needed to further investigate these sources as candidate cosmic ray and neutrino emitters. We report on the status of our investigation focused on blazars as candidate cosmic ray and neutrino emitters.
\end{abstract}

35th International Cosmic Ray Conference - ICRC2017

10-20 July, 2017

Bexco, Busan, Korea

${ }^{*}$ Speaker. 


\section{Introduction}

Blazars are Active Galactic Nuclei (AGN) hosting a jet oriented at a small angle with respect to the line of sight with highly relativistic particles moving in a magnetic field and emitting nonthermal radiation [1]. They are among the most luminous and most energetic persistent sources in the Universe. The radio to $\gamma$-ray spectral energy distributions (SEDs) of blazars are composed of two broad humps, a low energy and a high energy one. Blazars are subdivided in two main subclasses, namely flat-spectrum radio quasars (FSRQ) and BL Lacertae objects (BL Lacs), with the former displaying strong, broad emission lines and the latter instead being characterized by optical spectra showing at most weak emission lines, sometimes exhibiting absorption features, and in many cases being completely featureless. Based on the rest-frame value of the peak frequency of the low energy hump (synchrotron peak, $v_{S}$ ) blazars of the BL Lac type are called high energy peaked (HBLs) when $v_{S}>10^{15} \mathrm{~Hz}(>4 \mathrm{eV})$. HBLs are the rarest types of blazars, making up $\approx 10 \%$ of BL Lacs [2], being at the same time very powerful $\gamma$-ray emitters. The idea that blazars of various types could be sources of ultrahigh energy cosmic rays (CRs) and, related to that, of high energy neutrinos, has been discussed in e.g. $[3,4]$ and has since then been explored in a number of studies (see e.g. [5, 6, 7, 8, 9, 10]).

In 2015, the Fermi collaboration published the first high energy all-sky survey, the second catalog of hard Fermi-LAT sources (2FHL) [11]. The 360 sources composing the catalogue are detected in the $50 \mathrm{GeV}-2 \mathrm{TeV}$ energy range using data collected by the Fermi-Large Area Telescope (LAT) in 80 months of data (Pass 8).

The IceCube South Pole Neutrino Observatory has recently discovered high energy astrophysical neutrinos and has reported a sample of 54 events collected over a period of four years with a deposited energy up to $2 \mathrm{PeV}[12,13,14]$. These events are coming from the entire sky and make up what we define as the Isotropic Neutrino Emission ( $\mathrm{I} v \mathrm{E})$. They consist of neutrinos of all flavors, interacting within the instrumented volume (starting events), the majority of which are shower-like ${ }^{1}$. The complementary sample of through-going charged current $v_{\mu}$ from the northern sky has also been studied over a period of six years and recently been reported in [15] showing that their spectrum is inconsistent with the hypothesis of purely terrestrial origin at the $5.6 \sigma$ level. The track-like events confirm the general picture of the $\mathrm{I} v \mathrm{E}$ although their energy spectrum $\mathrm{E}^{-\gamma}$ slightly harder $(\gamma=2.13 \pm 0.13)$ with respect to the all sky one obtained from the starting event sample $(\gamma=2.58 \pm 0.25$ ), suggesting a mixed origin of the signal observed by IceCube. Many diverse hypothesis for the astrophysical counterparts of the $\mathrm{I} v \mathrm{E}$ have been put forward (see, e.g. $[16,17,18,19,20,21,22,23])$ but none has so far been statistically supported by the observational data.

The Pierre Auger Observatory [24, 25] and the Telescope Array (TA) [26] have collected together more than $300 \mathrm{CRs}$ with $E \geq 52 \times 10^{18} \mathrm{eV}$ over the entire sky. The former has detected 231 such events covering mostly the southern hemisphere and the latter 72 events above $57 \times 10^{18} \mathrm{eV}$ covering mostly the northern sky. Despite a series of studies on the CR arrival direction distributions [27, 28], no counterparts have yet been determined. The strongest deviations from isotropy

\footnotetext{
${ }^{1}$ Shower-like events are one of the topologies for neutrino observatories. Showers have a good energy resolution but reduced angular resolution $\left(\sim 10^{\circ}\right)$. The other topology are track-like events induced by muons, that have much better angular resolution $\left(<1^{\circ}\right)$, in which not all the energy is deposited on the detector.
} 
(post-trial probabilities $\sim 1.3-1.4 \%$ ) have been obtained for $E \geq 58 \times 10^{18} \mathrm{eV}$ in connection with Swift AGN closer than $130 \mathrm{Mpc}$ and more luminous than $10^{44} \mathrm{erg} \mathrm{s}^{-1}$, and around the direction of Cen A (at angular separations $\sim 18^{\circ}$ and $\sim 15^{\circ}$ respectively) [27]. The distribution of CRs does not present statistically significant small scale structures. However, two "hot" regions $(2-3 \sigma$ deviations) of about $20^{\circ}$ in the CR sky have been reported (see Fig. 1 top, [29], and [27]) but their interpretation is not yet clear.

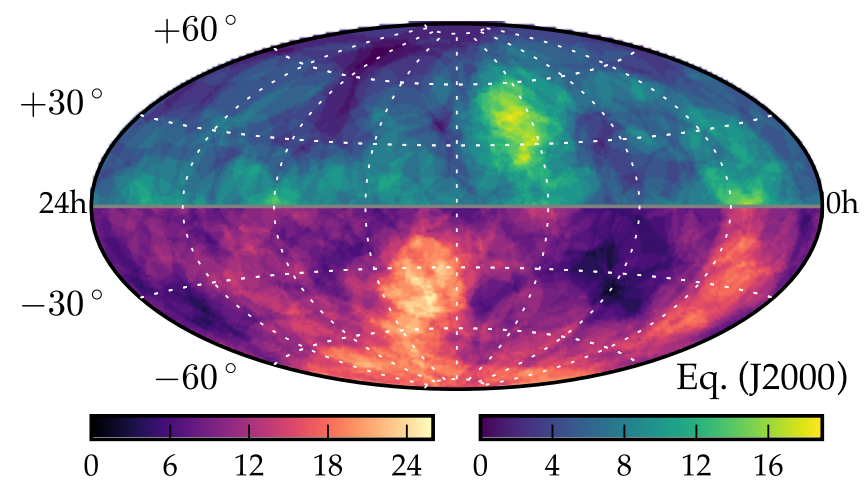

Figure 1: Sky maps in equatorial coordinates: Auger and TA data with uncertainty areas of $20^{\circ}$ as reported by the collaborations (and roughly the size of the two hot spots). The color scale indicates the number of events overlapping within the $20^{\circ}$ areas.

In [30], the three collaborations IceCube, Auger, and TA jointly reported about correlation tests between very high energy neutrinos and the same sample of CRs used in this paper. All the studies were compatible with the null hypothesis of no correlation although two interesting excesses were observed when considering IceCube shower-like events: a post-trial probability of $0.05 \%$ for an angular scale of $22^{\circ}$ provided by the comparison to a randomized CR sky, and a probability of $0.85 \%$ obtained by scrambling the neutrinos, thus preserving the existing anisotropies observed in the arrival directions of the CRs. As also discussed in [30], the first result (CR randomization), is affected by the presence of anisotropies (hot spots) in the CR sky (see Fig. 1). More interesting is the second result (neutrino randomization), which hints at a possible connection between IceCube neutrinos and CRs. An update of this study will be presented at this conference.

In [31] some of the authors have correlated the second catalogue of hard Fermi-LAT sources (2FHL) ( $E>50 \mathrm{GeV}, 360$ sources of various types, mainly blazars, [11]), the 2WHSP [32], and the 3LAC [33], with the publicly available high energy neutrino sample detected by IceCube ([13, $14,34,35]$, including only events with energy and median angular error $\left(\geq 60 \mathrm{TeV}\right.$ and $\leq 20^{\circ}$ respectively). The probability scanning over $F_{\gamma}(>50 \mathrm{GeV})$ was $0.4 \%$, which results in a global p-value of $1.4 \%(2.2 \sigma)$ after trial correction. This applies only to HBL blazars and appears to be strongly dependent on $\gamma$-ray flux.

Recently, we have extended the $\gamma$-ray sources to neutrinos connection and studied in [36] the possible blazar connection with CRs going through high energy neutrinos used as "intermediaries". We summarize here the method and the results reported in [36] updating them with the new data available. 
Motivated by the hints mentioned above on (1) an HBL origin of some of the IceCube neutrinos from [31], and (2) a common origin of neutrinos and CRs from [30], we have developed in [36] a two-step analysis to investigate the connection between $\gamma$-ray emitters and CRs.

\section{Neutrino filter to $\gamma$-ray sources $(v \rightarrow \gamma)$}

The neutrinos are implied here as filters in order to single out the best candidate lepto-hadronic accelerators [37] from $\gamma$-ray catalogs and hence the most probable CR sources. We have used in [36] all the high energy catalogs presently available including the 2FHL, the 2WHSP [32], and the 3LAC [33], as detailed in [31] focusing on HBL blazars ${ }^{2}$. In this paper we have updated the study using the 3FHL [38] and XXX. The neutrino list is composed of the 51 events (30 starting and 8 tracks) selected by [31] and the recently published 29 through going tracks reported in [15] of which one is a starting track also. No further catalogue has been tested and all the tests performed on the data are reported here not to hide any trial or relevant information.

As done in [31], we have filtered $\gamma$-ray sources in spatial coincidence with the neutrinos (i.e. within the median angular errors). The selection is done partitioning the flux of the cataloged sources $\left(F_{\gamma}\right)$ (or alternatively the "figure of merit" (FoM) introduced in the 2WHSP) ${ }^{3}$. Two subsets of the $\gamma$-ray catalogues are then obtained, the neutrino filtered and its complement:

$$
\begin{gathered}
(v \rightarrow \gamma) ; F_{\gamma} \text { neutrino filter } \\
\backslash(v \rightarrow \gamma) ; F_{\gamma} \text { complement. }
\end{gathered}
$$

\section{Neutrino filtered sources to $\mathbf{C R s}(v \rightarrow \gamma \rightarrow \mathbf{C R})$}

In a second independent step, the neutrino selected $\gamma$-ray sources at various $F_{\gamma}$ (or FoM) are cross correlated with the CRs. The correlation is also done as a function of the angular separation $\theta$ between the source and the reconstructed incoming direction of the CRs over the $1^{\circ}-30^{\circ}$ range in steps of $1^{\circ}$. This is because the CRs are charged particles and therefore deflected by an unknown angle due to the intervening magnetic fields.

We quantify the strength of the correlation by counting the number of CR events that have at least one neutrino filtered $\gamma$-ray source with $F_{\gamma}$ at distance smaller than the angular distance $\theta$ and compare this to random trials. This is done by randomizing the right ascension of the neutrino events and repeating the statistical analysis mentioned above. This randomization method is regularly used within the IceCube collaboration and ensures that the aforementioned anisotropies in the CRs are conserved and do not artificially contribute to the significance of the result, preserving at the same time the IceCube neutrino distribution, which is known to be not uniformly distributed.

The final probabilities are calculated based on random trials. The final result is corrected for trials due to the scanning in $F_{\gamma}$ (or FoM) and $\theta$ to search for the largest CR excess over the random

\footnotetext{
${ }^{2}$ The determination of $v_{S}$, which is required to classify sources as HBLs, requires the availability of multi-frequency data and is also affected by variability, a defining feature of blazars. The HBL - non-HBL distinction is therefore not sharp by definition.

${ }^{3}$ The FoM is defined as the ratio between the synchrotron peak flux of a source and that of the faintest blazar in the WHSP sample already detected in the TeV band.
} 
expectation. We have also corrected for the number of subsets in the $2 \mathrm{FHL}$ considering the relative correlations within the subsets. The additional factor is 2.9 .

Although the CR horizon is limited by the energy losses caused by the interactions of CRs with photons of the Cosmic Microwave Background (CMB), the so-called GZK (Greisen-ZatsepinKuzmin) effect $[39,40]$, we do not apply a-priori cuts on the distance of the cataloged sources. The reason is that a large number of BL Lacs has no measured redshift due to the lack of emission lines in their optical spectra. Moreover, it is difficult to quote a CR horizon a priori. Instead of scanning in redshift $z$ as done in other tests as the one in [27] and in [28], we use all sources regardless of their redshift.

As a last step, a likelihood ratio test comparing the connection between the filtered $(v \rightarrow \gamma)$ and the complement $\backslash(v \rightarrow \gamma)$ source samples with the CRs is performed. We define the test statistics as

$$
\Lambda=\frac{\mathscr{P}\left((\nu \rightarrow \gamma) ; F_{\gamma} \rightarrow C R ; \theta\right)}{\mathscr{P}\left(\backslash(\nu \rightarrow \gamma) ; F_{\gamma} \rightarrow C R ; \theta\right)}
$$

where $\mathscr{P}$ is the probability obtained in the two-step statistical test described above. Large values of $\Lambda$ indicate preference for a stronger correlation of neutrino filtered $\gamma$-ray emitters and CRs supporting a physical connection among the three astronomical messengers. We estimate the significance of this test by comparing data to trials with randomized neutrinos as discussed in the previous section. The p-value is then defined as the chance probability that trials produce a test statistics $\Lambda$ that is larger than, or equal to that observed.

\section{2FHL results}

The neutrino filter $(v \rightarrow \gamma)$ introduced in [31] selects the $\gamma$-ray emitters which are within the median angular errors of the IceCube neutrino events. A significant excess of CRs is here reported in connection with the neutrino filtered 2 FHL $\gamma$-ray emitters (see Fig. 2 solid lines). The $\left(F_{\gamma}, \theta\right)$ scan provides as the most significant excess a total of $83 \mathrm{CRs}$ at an angular separation $\theta<10^{\circ}$ from the 46 2FHL neutrino filtered $\gamma$-ray emitters with $F_{\gamma}(>50 \mathrm{GeV}) \geq 2.5 \times 10^{-11} \mathrm{phcm}^{-2} \mathrm{~s}^{-1}$. This number of CRs has to be compared with an expectation of 46.4 random associations determined on neutrino randomized cases. The probability of observing such an excess on randomized maps is $5.5 \times 10^{-5}$ which translates into $2.2 \times 10^{-3}$ after compensation for trials.

Among the subsets of $\gamma$-ray emitters reported for the 2FHL (4 nested subcatalogues reported by the FERMI collaboration), the best/minimal probability is obtained for the HBLs + unclassified sample. This sample includes all HBLs plus all sources with $2^{\circ}<\left|b_{\mathrm{II}}\right|<10^{\circ}$, which are still unclassified in the catalogue ${ }^{4}$. The scan values where the maximal excess is found are $F_{\gamma}(>50 \mathrm{GeV}) \geq$ $1.8 \times 10^{-11} \mathrm{ph} \mathrm{cm}^{-2} \mathrm{~s}^{-1}$ and $\theta=10^{\circ}$. At these scan values, a total of $80 \mathrm{CRs}$ are associated with the neutrino filtered sample, to be compared with an expectation of 42.5 random associations. The probability of observing such an excess on randomized maps is $2.4 \times 10^{-5}$ which translates into

\footnotetext{
${ }^{4}$ By excluding the Galactic plane, in fact, there is a high chance that these sources are unrecognized blazars lying in a region of the sky where the density of stellar sources is still quite high. Despite the fact that the optical counterparts of these $\gamma$-ray sources are still unknown, upon inspection, and after the test was carried out, it turned out that most of them have SEDs of the HBL type.
} 
$8.4 \times 10^{-4}(3.14 \sigma)$ after compensation for trials due to the $\left(F_{\gamma}, \theta\right)$ scan. Moreover, considering the fact that we have tested 4 nested subcatalogues of the $2 \mathrm{FHL}$, the final p-value is $2.4 \times 10^{-3}(2.8 \sigma)$.

No excess of CRs is found once the complement sample of $\gamma$-ray emitters, $\backslash(v \rightarrow \gamma)$, is considered (see Fig. 2 dotted lines). A likelihood ratio test $\Lambda$ (Eq. 3.1) comparing the connection between the filtered and the complement source samples with the CRs favours a connection between neutrino filtered emitters and CRs. With a p-value of $1.8 \times 10^{-4}(2.91 \sigma)$, the neutrino filter to CRs model is favoured providing a first strong hint of an association between HBLs and CRs. More statistics is required to confirm or disprove this scenario.
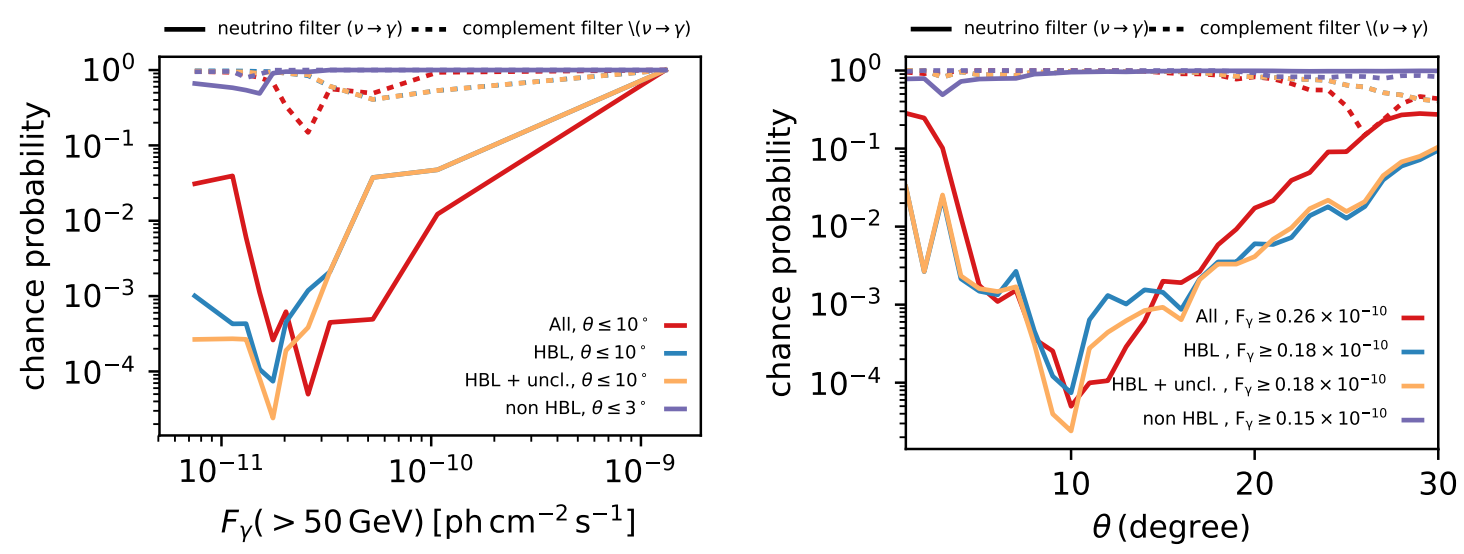

Figure 2: $(v \rightarrow \gamma \rightarrow \mathrm{CR})$ correlation test results (2FHL): (top) $F_{\gamma}$ projection of the chance probability at a fixed angular distance $\theta$. (bottom) $\theta$ projection of the chance probability at a fixed $F_{\gamma}$ value. The probabilities are reported for the neutrino filtered $\gamma$-ray emitters (solid line) and for the complement sample (dashed line) at $F_{\gamma}$ (or $\theta$ ) larger than the value on the x-axis.

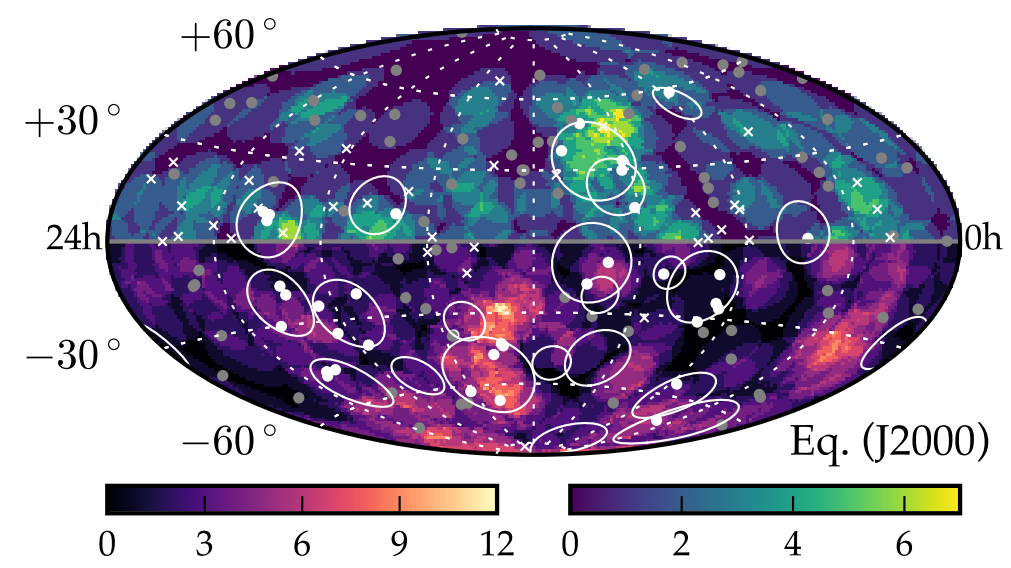

Figure 3: Sky maps in equatorial coordinates: Auger and TA data with uncertainty areas of $10^{\circ}$ as obtained from our test with overlaid neutrinos contributing to the test (crosses are track-like events, circles are showerlike events with associated median angular error). Dots indicate the $2 \mathrm{FHL}$ (HBL + unclassified sample) objects that are filtered (white) or not filtered (grey) by neutrinos as described in the text. 


\section{References}

[1] C. M. Urry and P. Padovani, Publ. Astron. Soc. Pac. 107 (1995) 803.

[2] P. Padovani and P. Giommi, Astrophys. J. 444 (1995) 567.

[3] K. Mannheim, Astropart. Phys. 3 (1995) 295-302.

[4] F. Halzen and E. Zas, Astrophys. J. 488 (1997) 669-674.

[5] A. Muecke, R. J. Protheroe, R. Engel, J. P. Rachen, and T. Stanev, Astropart. Phys. 18 (2003) 593-613.

[6] M. D. Kistler, T. Stanev, and H. Yüksel, Phys. Rev. D90 (2014) 123006.

[7] K. Murase, Y. Inoue, and C. D. Dermer, Phys. Rev. D90 (2014) 023007.

[8] F. Tavecchio and G. Ghisellini, Mon. Not. Roy. Astron. Soc. 451 (2015) 1502-1510.

[9] P. Padovani, M. Petropoulou, P. Giommi, and E. Resconi, Mon. Not. Roy. Astron. Soc. 452 (2015) 1877-1887.

[10] M. D. Kistler and R. Laha, ArXiv e-prints (May, 2016).

[11] Fermi-LAT Collaboration, M. Ackermann et al., Astrophys. J. Suppl. 222 (2016) 5.

[12] IceCube Collaboration, M. G. Aartsen et al., Science 342 (2013) 1242856.

[13] IceCube Collaboration, M. G. Aartsen et al., Phys. Rev. Lett. 113 (2014) 101101.

[14] IceCube Collaboration, M. G. Aartsen et al., ArXiv e-prints (Oct., 2015).

[15] IceCube Collaboration, M. G. Aartsen et al., Astrophys. J. 833 (2016) 3.

[16] B. Khiali and E. M. de Gouveia Dal Pino, MNRAS 455 (Jan., 2016) 838-845.

[17] R. Moharana, R. J. G. Britto, and S. Razzaque, ArXiv e-prints (Feb., 2016).

[18] K. Murase, D. Guetta, and M. Ahlers, Physical Review Letters 116 (Feb., 2016) 071101.

[19] I. Tamborra and S. Ando, Phys. Rev. D 93 (Mar., 2016) 053010.

[20] N. Senno, K. Murase, and P. Mészáros, Phys. Rev. D 93 (Apr., 2016) 083003.

[21] X.-Y. Wang and R.-Y. Liu, Phys. Rev. D 93 (Apr., 2016) 083005.

[22] V. N. Zirakashvili and V. S. Ptuskin, Astroparticle Physics 78 (May, 2016) 28-34.

[23] A. Neronov and D. Semikoz, Phys. Rev. D 93 (June, 2016) 123002.

[24] Pierre Auger Collaboration, J. Abraham et al., Nucl. Instrum. Meth. A523 (2004) 50-95.

[25] Pierre Auger Collaboration, A. Aab et al., Nucl. Instrum. Meth. A798 (2015) 172-213.

[26] Telescope Array Collaboration, T. Abu-Zayyad et al., Nucl. Instrum. Meth. A689 (2013) 87-97.

[27] Pierre Auger Collaboration, A. Aab et al., Astrophys. J. 804 (2015) 15.

[28] Telescope Array Collaboration, T. Abu-Zayyad et al., Astrophys. J. 777 (2013) 88.

[29] Telescope Array Collaboration, R. U. Abbasi et al., Astrophys. J. 790 (2014) L21.

[30] IceCube, Pierre Auger, Telescope Array Collaboration, M. G. Aartsen et al., JCAP 1601 (2016) 037.

[31] P. Padovani, E. Resconi, P. Giommi, B. Arsioli, and Y. L. Chang, Mon. Not. Roy. Astron. Soc. 457 (2016) 3582-3592. 
[32] Y.-L. Chang, B. Arsioli, P. Giommi, and P. Padovani, Astron. Astrophys., in press (2017).

[33] Fermi-LAT Collaboration, M. Ackermann et al., Astrophys. J. 810 (2015) 14.

[34] IceCube Collaboration, M. G. Aartsen et al., Phys. Rev. Lett. 115 (2015) 081102.

[35] IceCube Collaboration, S. Schoenen and L. Raedel, The Astronomer's Telegram 7856 (July, 2015).

[36] E. Resconi, S. Coenders, P. Padovani, P. Giommi, and L. Caccianiga, Mon. Not. Roy. Astron. Soc. 468 (2017) 597.

[37] M. Petropoulou, S. Dimitrakoudis, P. Padovani, A. Mastichiadis, and E. Resconi, Mon. Not. Roy. Astron. Soc. 448 (2015) 2412-2429.

[38] Fermi-LAT Collaboration, arXiv:1702.00664.

[39] K. Greisen, Phys. Rev. Lett. 16 (1966) 748-750.

[40] G. T. Zatsepin and V. A. Kuzmin, JETP Lett. 4 (1966) 78-80. [Pisma Zh. Eksp. Teor. Fiz.4,114(1966)]. 